\title{
CHAOTIFYING 2-D PIECEWISE-LINEAR MAPS VIA A PIECEWISE-LINEAR CONTROLLER FUNCTION
}

\author{
Z. Elhadj ${ }^{1}$ and J.C. Sprott ${ }^{2}$
}

UDC 517.9

\begin{abstract}
A simple method for chaotifying piecewise-linear maps of the plane using a piecewise-linear controller function is given. A domain of chaos in the resulting controlled map was determined exactly and rigorously.
\end{abstract}

\section{Introduction}

A large number of physical and engineering systems have been found to be governed by a class of continuous or discontinuous maps $[1,4,5,7-10,13,14]$ where the discrete-time state space is divided into two or more compartments with different functional forms of the map separated by borderlines [14-17]. The theory of discontinuous maps is in its infancy, with some progress reported for 1-D and $n$-D discontinuous maps in $[1,8,9,11,12$, 17], but these results are restrictive and cannot be obtained in the general $n$-dimensional context [12]. On the other hand, there are many works that focus on the chaotic behavior of discrete mappings. For example, they have been studied as control and anticontrol (chaotification) schemes using Lyapunov exponents $[2,3,18,20]$ to prove the existence of chaos in $n$-dimensional discrete dynamical systems with the goal of making in some way an originally nonchaotic dynamical system chaotic or enhancing the chaos already existing in such a system.

The goal of this work is to present a simple method for chaotifying an arbitrary 2-D piecewise-linear map (continuous or not) using a simple piecewise-linear controller function, which allows one to determine exactly and rigorously which portion of the bifurcation parameter space is characterized by the occurrence of chaos in the resulting controlled map using the standard definition of the Lyapunov exponents as the test for chaos.

Consider an arbitrary piecewise-linear map $f: D \rightarrow D$, where $D=D_{1} \cup D_{2} \subset \mathbb{R}^{2}$, defined by

$$
X_{k+1}=f\left(X_{k}\right)=\left\{\begin{array}{lll}
A X_{k}+b & \text { if } & X_{k} \in D_{1}, \\
B X_{k}+c & \text { if } & X_{k} \in D_{2}
\end{array}\right.
$$

where

$$
A=\left(\begin{array}{ll}
a_{11} & a_{12} \\
a_{21} & a_{22}
\end{array}\right) \quad \text { and } \quad B=\left(\begin{array}{ll}
b_{11} & b_{12} \\
b_{21} & b_{22}
\end{array}\right)
$$

are $2 \times 2$ real matrices,

$$
b=\left(\begin{array}{l}
b_{1} \\
b_{2}
\end{array}\right) \quad \text { and } \quad c=\left(\begin{array}{l}
c_{1} \\
c_{2}
\end{array}\right)
$$

\footnotetext{
${ }^{1}$ University of Tébessa, Tébessa, Algeria; e-mail: zeraoulia@mail.univ-tebessa.dz, zelhadj12@yahoo.fr.

${ }^{2}$ University of Wisconsin-Madison, Madison, USA; e-mail: sprott@ physics.wisc.edu.
}

Published in Neliniini Kolyvannya, Vol. 13, No.3, pp. 328-335, July-September, 2010. Original article submitted December 9, 2008; revision submitted April 15, 2009. 
are $2 \times 1$ real vectors, and

$$
X_{k}=\left(\begin{array}{c}
x_{k} \\
y_{k}
\end{array}\right) \in \mathbb{R}^{2}
$$

is the state variable.

The Lyapunov exponents of a 2-D dynamical system are defined as follows:

Consider the system

$$
X_{k+1}=f\left(X_{k}\right), \quad X_{k} \in \mathbb{R}^{2}, \quad k=0,1,2, \ldots,
$$

where the function $f: \mathbb{R}^{2} \longrightarrow \mathbb{R}^{2}$ is the vector field associated with system (2). Let $J\left(X_{k}\right)$ be its Jacobian evaluated at $X_{k} \in \mathbb{R}^{2}, k=0,1,2, \ldots$, and define the matrix

$$
T_{n}\left(X_{0}\right)=J\left(X_{n-1}\right) J\left(X_{n-2}\right) \ldots J\left(X_{1}\right) J\left(X_{0}\right) .
$$

Moreover, let $J_{i}\left(X_{0}, n\right)$ be the modulus of the $i$ th eigenvalue of the $n$th matrix $T_{n}\left(X_{0}\right)$, where $i=1,2$ and $n=0,1,2, \ldots$.

The Lyapunov exponents for a two-dimensional discrete-time system are now defined by

$$
l_{i}\left(X_{0}\right)=\lim _{n \rightarrow+\infty} \ln \left(J_{i}\left(X_{0}, n\right)^{\frac{1}{n}}\right), \quad i=1,2
$$

Roughly speaking, chaotic behavior implies sensitive dependence on initial conditions, with at least one positive Lyapunov exponent. Based on this definition, we give in the next section a rigorous proof of chaos in the resulting controlled map (7) obtained below via a simple piecewise-linear controller function applied to map (1). While many algorithms for calculating the Lyapunov exponents would give spurious results for piecewise-linear discontinuous maps, the algorithm used here and given in [21] works for such cases. It essentially takes a numerical derivative and gives the correct result provided that is taken to ensure that the perturbed and unperturbed orbits lie on the same side of the discontinuity. This may require an occasional small perturbation into a region that is not strictly accessible to the orbit.

\section{Chaotification Method Using a Piecewise-Linear Controller Function}

The main idea of the chaotification method presented in this work is to introduce a piecewise-linear controller function in such a way that the two (in both partitions) system matrices of the resulting controlled piecewise-linear system have the same trace and determinant (invariants) and, hence, the same eigenvalues. Indeed, the controlled map is given by

$$
g\left(X_{k}\right)=\left\{\begin{array}{lll}
A X_{k}+b & \text { if } & X_{k} \in D_{1} \\
B X_{k}+c & \text { if } & X_{k} \in D_{2}
\end{array}+U\left(X_{k}\right),\right.
$$

where the controller function $U\left(X_{k}\right)$ is defined by 


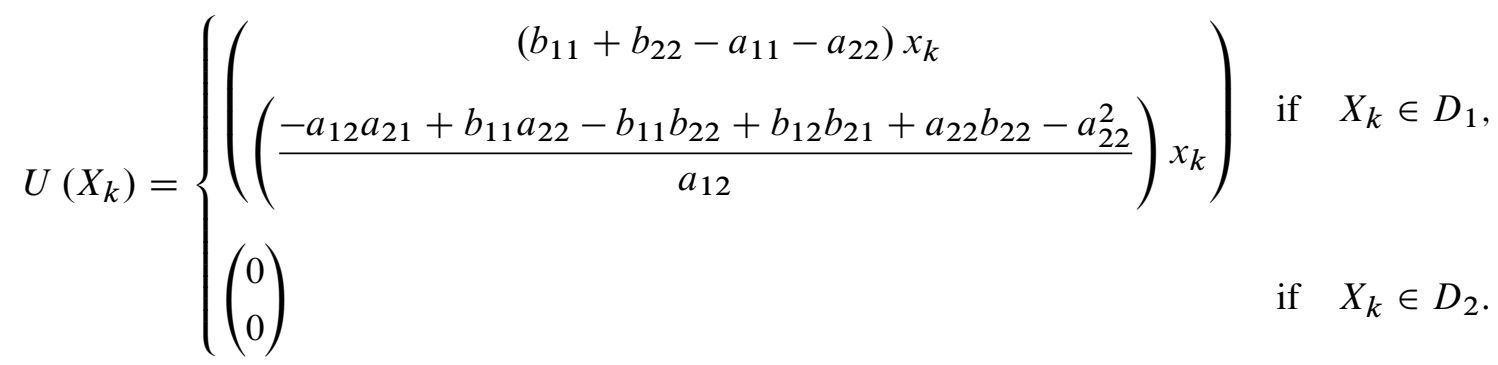

The controlled system (5) is now given by

$$
g\left(X_{k}\right)=\left\{\begin{array}{lll}
Q X_{k}+b & \text { if } & X_{k} \in D_{1}, \\
B X_{k}+c & \text { if } & X_{k} \in D_{2},
\end{array}\right.
$$

where the matrix $Q$ is given by

$$
Q=\left(\begin{array}{cc}
b_{11}+b_{22}-a_{22} & a_{12} \\
\frac{b_{11} a_{22}-b_{11} b_{22}+b_{12} b_{21}+a_{22} b_{22}-a_{22}^{2}}{a_{12}} & a_{22}
\end{array}\right) .
$$

The Jacobian matrix of the controlled map (7) is given by

$$
J\left(X_{k}\right)=\left\{\begin{array}{lll}
Q & \text { if } & X_{k} \in D_{1} \\
B & \text { if } & X_{k} \in D_{2}
\end{array}\right.
$$

It is shown in [22] that if we consider a system $x_{k+1}=f\left(x_{k}\right), x_{k} \in \Omega \subset \mathbb{R}^{n}$, such that

$$
\left\|f^{\prime}(x)\right\|=\sqrt{\lambda_{\max }\left(f(x)^{T} f(x)\right)} \leq N<+\infty,
$$

with a smallest eigenvalue of $f(x)^{T} f(x)$ that satisfies

$$
\lambda_{\min }\left(f^{\prime}(x)^{T} f^{\prime}(x)\right) \geq \theta>0,
$$

where $N^{2} \geq \theta$, then, for any $x_{0} \in \Omega$, all the Lyapunov exponents at $x_{0}$ are located inside $\left[\frac{\ln \theta}{2}, \ln N\right]$, i.e.,

$$
\frac{\ln \theta}{2} \leq l_{i}\left(x_{0}\right) \leq \ln N, \quad i=1,2, \ldots, n,
$$

where $l_{i}\left(x_{0}\right)$ are the Lyapunov exponents for the map $f$ and $\|\cdot\|$ is the Euclidean norm in $\mathbb{R}^{n}$. We remark that $J\left(X_{k}\right)$ given in (9) is not well defined due to the discontinuity, but, since $B$ and $Q$ have the same eigenvalues, 
one has $\|Q\|=\|B\|=\sqrt{\lambda_{\max }\left(B^{T} B\right)}$. Since

$$
B^{T} B=\left(\begin{array}{cc}
b_{11}^{2}+b_{21}^{2} & b_{11} b_{12}+b_{21} b_{22} \\
b_{11} b_{12}+b_{21} b_{22} & b_{12}^{2}+b_{22}^{2}
\end{array}\right)
$$

is at least a positive semidefinite matrix, all its eigenvalues are real and positive, i.e.,

$$
\lambda_{\max }\left(B^{T} B\right) \geq \lambda_{\min }\left(B^{T} B\right) \geq 0 .
$$

Hence, the eigenvalues of $B^{T} B$ are given by

$$
\begin{aligned}
& \lambda_{\max }\left(B^{T} B\right)=\frac{1}{2} b_{11}^{2}+\frac{1}{2} b_{12}^{2}+\frac{1}{2} b_{21}^{2}+\frac{1}{2} b_{22}^{2}+\frac{1}{2} \sqrt{d}, \\
& \lambda_{\min }\left(B^{T} B\right)=\frac{1}{2} b_{11}^{2}+\frac{1}{2} b_{12}^{2}+\frac{1}{2} b_{21}^{2}+\frac{1}{2} b_{22}^{2}-\frac{1}{2} \sqrt{d},
\end{aligned}
$$

where

$$
d=\left(\left(b_{11}+b_{22}\right)^{2}+\left(b_{12}-b_{21}\right)^{2}\right)\left(\left(b_{12}+b_{21}\right)^{2}+\left(b_{11}-b_{22}\right)^{2}\right)>0
$$

for all $b_{11}, b_{12}, b_{21}$, and $b_{22}$. Condition (10) gives

$$
\left\|f^{\prime}(x)\right\|=\|B\|=\|Q\|=\sqrt{\lambda_{\max }\left(B^{T} B\right)}=N<+\infty
$$

because $B$ and $Q$ have the same eigenvalues. Condition (11) gives the inequality

$$
\theta^{2}-\left(b_{11}^{2}+b_{12}^{2}+b_{21}^{2}+b_{22}^{2}\right) \theta+\left(b_{11} b_{22}-b_{12} b_{21}\right)^{2} \geq 0
$$

with the condition

$$
\theta<\frac{b_{11}^{2}+b_{12}^{2}+b_{21}^{2}+b_{22}^{2}}{2}
$$

Since the discriminant of (15) is equal to $d>0$, (11) holds if

$$
\theta \geq \lambda_{\max }\left(B^{T} B\right) \quad \text { or } \quad \theta \leq \lambda_{\min }\left(B^{T} B\right)
$$

The condition $\theta \geq \lambda_{\max }\left(B^{T} B\right)$ is impossible because of condition (16), so that $\theta$ must satisfy the condition

$$
\theta<\lambda_{\min }\left(B^{T} B\right)=\frac{b_{11}^{2}+b_{12}^{2}+b_{21}^{2}+b_{22}^{2}-\sqrt{d}}{2} .
$$


Now, if

$$
\begin{gathered}
2<b_{11}^{2}+b_{12}^{2}+b_{21}^{2}+b_{22}^{2}<\left(b_{11} b_{22}-b_{12} b_{21}\right)^{2}+1, \\
\left|b_{11} b_{22}-b_{12} b_{21}\right|>1,
\end{gathered}
$$

then $\lambda_{\min }\left(B^{T} B\right)>1$, i.e., $\theta=1$, and one has

$$
0<l_{i}\left(x_{0}\right) \leq \ln N, \quad i=1,2,
$$

i.e., the controlled map (7) converges to a hyperchaotic attractor for all parameters $b_{11}, b_{12}, b_{21}$, and $b_{22}$ satisfying (19).

Finally, the following theorem is proved:

Theorem 1. The piecewise-linear controller function (6) makes map (1) chaotic in the following case:

$$
\begin{gathered}
2<b_{11}^{2}+b_{12}^{2}+b_{21}^{2}+b_{22}^{2}<\left(b_{11} b_{22}-b_{12} b_{21}\right)^{2}+1, \\
\left|b_{11} b_{22}-b_{12} b_{21}\right|>1 .
\end{gathered}
$$

Finally, we note that Theorem 1 does not guarantee the boundedness of the resulting controlled map (7). This problem is still open for general piecewise-linear maps and flows.

\section{Example}

In this section, we make chaotic the following piecewise-linear map using the above method:

$$
f\left(x_{k}, y_{k}\right)=\left\{\begin{array}{c}
\left(\begin{array}{c}
x_{k}-\alpha y_{k}+1, \\
\gamma x_{k}
\end{array}\right) \quad \text { if } \quad y_{k} \geq 0, \\
\left(\begin{array}{c}
x_{k}+\alpha y_{k}+1 \\
-\beta x_{k}
\end{array}\right) \quad \text { if } \quad y_{k}<0,
\end{array}\right.
$$

where $\alpha, \beta$, and $\gamma$ are bifurcation parameters. Map (22) is a special case of map (1) because one can rewrite it as

$$
f\left(X_{k}\right)=\left\{\begin{array}{llc}
A X_{k}+b & \text { if } & y_{k} \geq 0 \\
B X_{k}+c & \text { if } \quad y_{k}<0
\end{array}\right.
$$

where

$$
A=\left(\begin{array}{cc}
1 & -\alpha \\
\gamma & 0
\end{array}\right), \quad B=\left(\begin{array}{cc}
1 & \alpha \\
-\beta & 0
\end{array}\right), \quad b=c=\left(\begin{array}{l}
1 \\
0
\end{array}\right),
$$




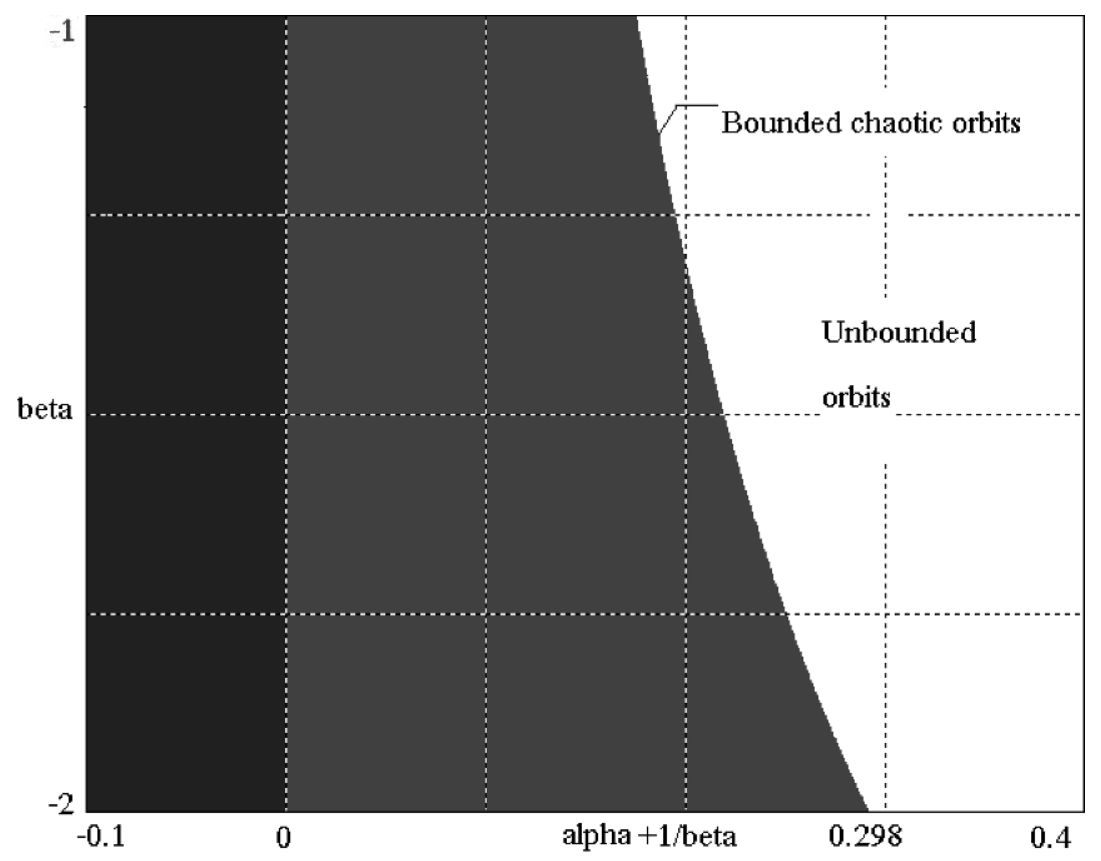

Fig. 1. Regions of dynamical behaviors in the -plane for the controlled map (23) with 2 $2<1$ and $0.11 /<<$ $1 /+0.4$.

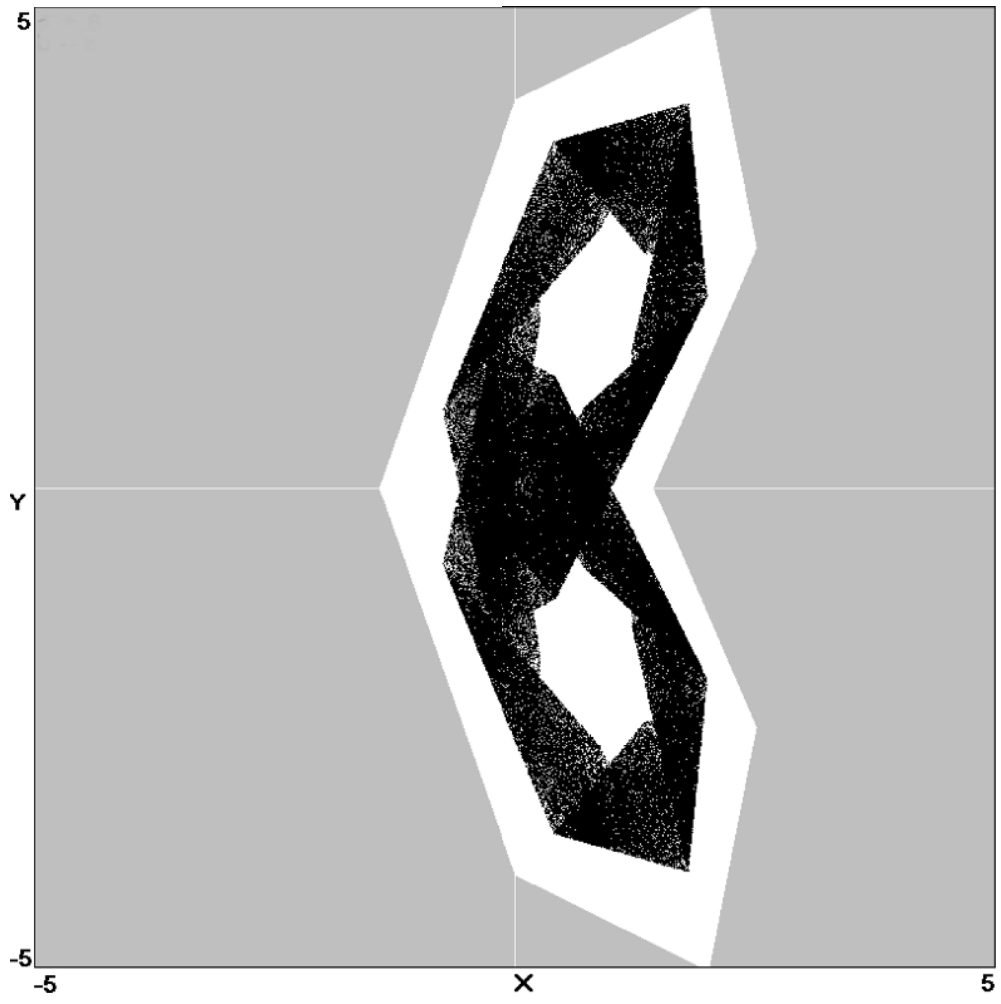

Fig. 2. The new piecewise-linear chaotic attractor obtained from the controlled map (23) with its basin of attraction for $=0.6$, $=2$, and the initial condition $x_{0}=y_{0}=0.01$. 


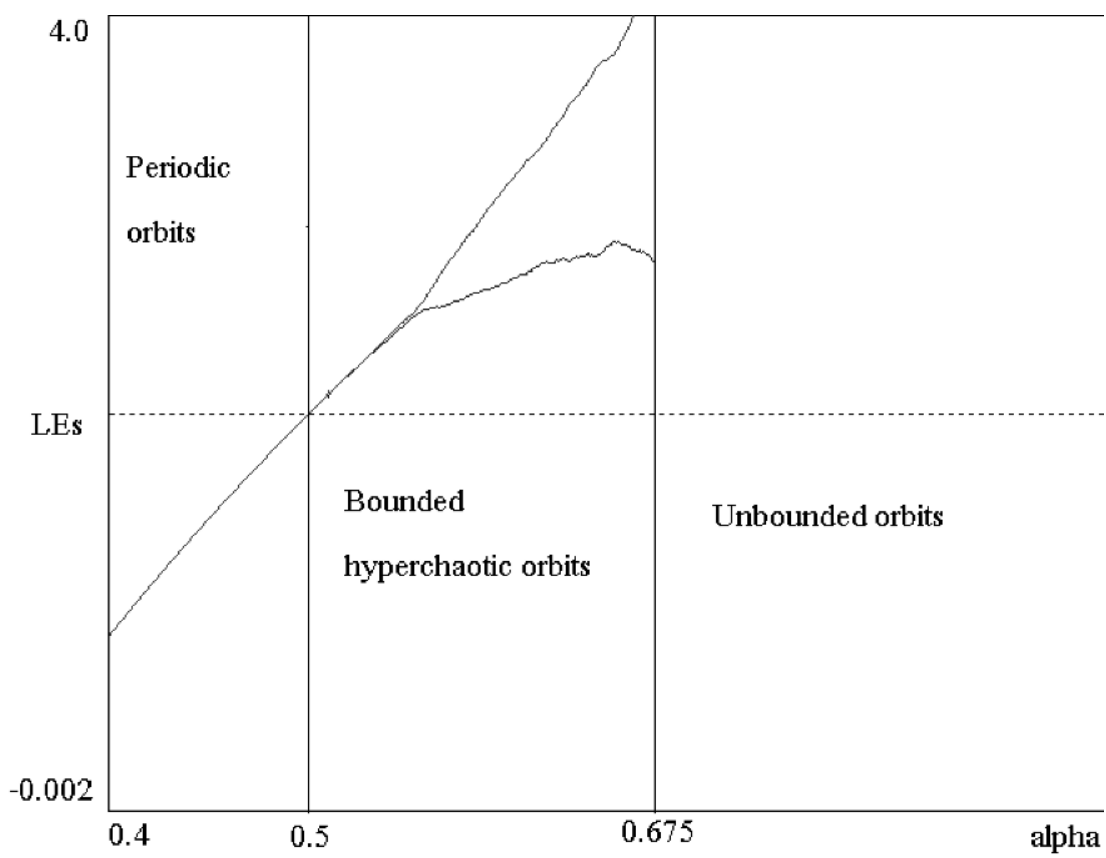

Fig. 3. Variation of the Lyapunov exponents of the controlled map (23) versus the parameter $0.4 \leq \alpha \leq 0.9$, with $\beta=-2$, where the two vertical lines indicate the border between the mentioned dynamical behaviors.

and the two subregions are $D_{1}=\left\{\left(x_{k}, y_{k}\right) \in \mathbb{R}^{2} / y_{k} \geq 0\right\}$ and $D_{2}=\left\{\left(x_{k}, y_{k}\right) \in \mathbb{R}^{2} / y_{k}<0\right\}$. Thus, the resulting controlled map is given by

$$
g\left(x_{k}, y_{k}\right)=\left\{\begin{array}{l}
\left(\begin{array}{cc}
1 & -\alpha \\
\beta & 0
\end{array}\right)\left(\begin{array}{l}
x_{k} \\
y_{k}
\end{array}\right)+\left(\begin{array}{l}
1 \\
0
\end{array}\right) \quad \text { if } \quad y_{k} \geq 0 \\
\left(\begin{array}{cc}
1 & \alpha \\
-\beta & 0
\end{array}\right)\left(\begin{array}{l}
x_{k} \\
y_{k}
\end{array}\right)+\left(\begin{array}{l}
1 \\
0
\end{array}\right) \quad \text { if } \quad y_{k}<0
\end{array}=\left(\begin{array}{c}
1-\alpha\left|y_{k}\right|+x_{k} \\
\beta x_{k} \operatorname{sgn}\left(y_{k}\right)
\end{array}\right),\right.
$$

which is the so-called discrete butterfly presented in [19], where $\operatorname{sgn}(\cdot)$ is the standard signum function that gives \pm 1 depending on the sign of its argument. For the controlled map (23), condition (21) becomes

$$
|\alpha|>\max \left(\frac{|\beta|}{\sqrt{\beta^{2}-1}}, \frac{1}{|\beta|}\right), \quad|\beta|>1
$$

For illustration, assume that $\beta<-1$. Then we remark that

$$
\frac{|\beta|}{\sqrt{\beta^{2}-1}}=\frac{-\beta}{\sqrt{\beta^{2}-1}}<\frac{1}{|\beta|}=\frac{-1}{\beta},
$$


and, thus, conditions (24) become

$$
|\alpha|>\frac{-1}{\beta}, \quad \beta<-1
$$

Using the obtained analytical results, Fig. 1 shows that, for

$$
-2 \leq \beta<-1 \quad \text { and } \quad-0.1-\frac{1}{\beta}<\alpha<\frac{-1}{\beta}+0.4
$$

the controlled map (23) converges to bounded hyperchaotic attractors or unbounded orbits for $\alpha>-\frac{1}{\beta}$. In this figure, unbounded solutions, periodic solutions, and chaotic solutions are shown in the $\alpha \beta$-plane for the controlled map (23), where we use 5000 different initial conditions and $10^{6}$ iterations for each point. A chaotic attractor for the case with $\alpha=0.6$ and $\beta=-2$ is shown in Fig. 2.

On the other hand, it is necessary to verify the hyperchaoticity of the attractors by calculating both Lyapunov exponents using the formula $l_{1}\left(x_{0}\right)+l_{2}\left(x_{0}\right)=\ln |\operatorname{det}(J)|=\ln |\alpha \beta|$ averaged along the orbit, where $\operatorname{det}(J)$ is the determinant of the Jacobian matrix. The result is shown in Fig. 3 for $0.4 \leq \alpha \leq 0.9$, with $\beta=-2$.

\section{Conclusion}

A new simple chaotification method for piecewise-linear maps of the plane via a piecewise-linear controller function was presented. A rigorous proof of chaos in the resulting controlled map using the standard definition of the largest Lyapunov exponent was also given.

\section{REFERENCES}

1. A. N. Sharkovsky and L. O. Chua, "Chaos in some 1-D discontinuous maps that appear in the analysis of electrical circuits," IEEE Trans. Circuits Syst., 40, 722-731 (1993).

2. C. Li, "On super-chaotifying discrete dynamical systems," Chaos, Solitons Fractals, 21, 855-861 (2004).

3. G. Chen and D. Lai, "Making a discrete dynamical system chaotic: feedback control of Lyapunov exponents for discrete-time dynamical system," IEEE Trans. Circuits Syst.-I, 44, 250-253 (1997).

4. G. H. Yuan, S. Banerjee, E. Ott, and J. A. Yorke, "Border collision bifurcations in the buck converter," IEEE Trans. Circuits Systems-I, 45, 707-716 (1998).

5. G. M. Maggio, M. di Bernardo, and M.P. Kennedy, "Nonsmooth bifurcations in a piecewise-linear model of the Colpitts oscillator," IEEE Trans. Circuits Syst.-I, 8, 1160-1177 (2000).

6. L. E. Kollar, G. Stepan, and J. Turi, "Dynamics of piecewise linear discontinuous maps," Int. J. Bifurcation Chaos, 14, 2341-2351 (2004).

7. L. O. Chua and T. Lin, "Chaos in digital filters," IEEE Trans. Circuits Syst., 35, 648-658 (1988).

8. M. J. Ogorzalek, “Complex behavior in digital filters," Int. J. Bifurcation Chaos, 2, No. 1, 11-29 (1992).

9. O. Feely and L. O. Chua, "Nonlinear dynamics of a class of analog-to-digital converters," Int. J. Bifurcation Chaos, 22, 325-340 (1992).

10. O. Feely and L. O. Chua, "The effect of integrator leak in modulation," IEEE Trans. Circuits Syst-I, 38, 1293-1305 (1991).

11. P. Jain and S. Banerjee, "Border collision bifurcations in one-dimensional discontinuous maps," Int. J. Bifurcation Chaos, 13, 33413352 (2003).

12. P. S. Dutta, B. Routroy, S. Banerjee, and S. S. Alam, "Border collision bifurcations in $n$-dimensional piecewise linear discontinuous maps," Chaos (to appear).

13. R. Rajaraman, I. Dobson, and S. Jalali, "Nonlinear dynamics and switching time bifurcations of a thyristor controlled reactor circuit," IEEE Trans. Circuits Syst.-I, 43, 1001-1006 (1996).

14. S. Banerjee and G. C. Verghese (editors), Nonlinear Phenomena in Power Electronics: Attractors, Bifurcations, Chaos, and Nonlinear Control, IEEE Press, New York (2001). 
15. S. Banerjee, E. Ott, J. A. Yorke, and G. H. Yuan, "Anomalous bifurcations in dc-dc converters: borderline collisions in piecewise smooth maps," in: Proceedings of IEEE Power Electronics Specialists' Conference (1997), pp. 1337-1344.

16. S. Banerjee, S. Parui, and A. Gupta, "Dynamical effects of missed switching in current-mode controlled dc-dc converters," IEEE Trans. Circuits Syst.-II, 51, 649-654 (2004).

17. T. K. Tse, Complex Behavior of Switching Power Converters, CRC Press, Boca Raton (2003).

18. X. F. Wang and G. Chen, "On feedback anticontrol of discrete chaos," Int. J. Bifurcation Chaos, 9, 1435-1441 (1999).

19. Z. Elhadj, "The discrete butterfly," Chaos, Solitons Fractals (to appear).

20. Z. Li, J. B. Park, Y. H. Joo, Y. H. Choi, and G. Chen, "Anticontrol of chaos for discrete TS fuzzy systems," IEEE Trans. Circuits Syst.-I, 49, 249-253 (2002).

21. J. C. Sprott, Chaos and Time-Series Analysis, Oxford University Press, Oxford (2003).

22. C. Li and G. Chen, "Estimating the Lyapunov exponents of discrete systems," Chaos, 14, No. 2, 343-346 (2000). 